\title{
Content-Based Image Unequal Error Protection Strategies for an Open Loop MIMO System
}

\author{
Rebeca Colda $^{1}$, Clency Perrine ${ }^{2}$, Jean-Pierre Cances ${ }^{3}$, Rodolphe Vauzelle ${ }^{2}$, Tudor Palade ${ }^{1}$ \\ ${ }^{1}$ Communications Department, Faculty of Electronics, Telecommunications and Information Technology, \\ Technical University of Cluj-Napoca, Cluj-Napoca, Romania \\ ${ }^{2}$ Signal Image and Communications Department, XLIM Laboratory, University of Poitiers, Poitiers, France \\ ${ }^{3}$ Components Circuits Signals and High Frequency Systems Department, XLIM Laboratory, \\ University of Limoges, Limoges, France \\ Email: rebeca.colda@com.utcluj.ro, clency.perrine@univ-poitiers.fr
}

Received September 20, 2011; revised October 29, 2011; accepted November 13, 2011

\begin{abstract}
The paper deals with strategies for optimizing the transmission of JPEG2000 coded images over a MIMO quasi-static Rayleigh fading channel, by exploiting different available MIMO STBC (Space Time Block Codes), as well as the image content to be transmitted. The aim is to propose a link adaptation scheme based on the variable radio channel's conditions, by establishing an UEP (Unequal Error Protection) scheme that leads to the best tradeoff between the robustness to errors and the image visual quality. Comparisons are made with the EEP (Equal Error Protection) case, in terms of the PSNR (Peak Signal to Noise Ratio) fidelity metric, as well as the subjective image quality.
\end{abstract}

Keywords: EEP; UEP; MIMO STBCs; JPEG2000 Wireless (JPWL)

\section{Introduction}

Nowadays, multimedia wireless applications have become very popular mainly due to the advances of wireless technologies that are now able to offer high data rates to both stationary and mobile users. As the available spectrum resources are limited, compression techniques are employed in order to remove the amount of redundant data, thus making the code-stream more sensitive to transmission errors.

On the other hand, due to the unstable nature of the wireless channel that induces frequent transmission errors, error resilience mechanisms must be employed for assuring at least a basic image quality even under a worst case scenario. Thus, the design of efficient radio resource management schemes for assuring an optimal error protection has become a challenging issue. The problem of reliable transmission of JPEG2000 coded images over error-prone wireless channels is addressed in the 11th amendment of the JPEG2000 standard, called JPWL (JPEG2000 Wireless) [1], where a framework for error protection and correction is defined.

Unlike JPEG2000 baseline, JPWL provides tools for dealing with the protection of the image header, the most sensitive part of the whole code-stream, data partitioning and interleaving as well as UEP (Unequal Error Protection) of the data according to its importance to the overall image quality. For applying UEP, different FEC
(Forward Error Correction) codes can be employed, such as Reed-Solomon codes, but the difficulty relies in deciding which data are essential with respect to the image quality and which data can be transmitted with less reliability [2].

On the other hand, the benefits of the MIMO (Multiple Input Multiple Output) technology, in terms of increased data rates or increased reliability, over SISO (Single Input Single Output) systems are well known [3]. In this paper, the case of a MIMO-OL (Open Loop) system is considered, where the channel state information is known only at the receiver side (CSIR). Thus, at the transmitter side the only adaptation possible results from exploiting the source content by applying a proper joint sourcechannel coding scheme.

The main novelty of this paper consists in showing the importance and the gain that results both from the ex ploitation of the content to be transmitted in a MIMO-OL system as well as the error performance of different available STBC (Space Time Block Coding) techniques. It will be shown that the reliability of a certain channel protection scheme can be increased not only by applying an unequal error protection of the data (UEP), but even more by combining it with a suitable STC (Space Time Coding) technique. On the other hand, by taking into account the content to be transmitted, significant improvements with respect to the image quality can be ob- 
tained even for the case of a diminished error performance STBC.

Different UEP strategies are proposed in the literature as in [4], but in most of them the channel is considered to be a memory-less Binary Symmetrical one. The assumption of such a channel affected by uncorrelated errors is not a realistic one, because usually the wireless channel is affected by bursts of errors that have an impact upon several sequences of bits.

In other papers, as in [5] and [6], adaptive UEP schemes are proposed on Rayleigh fading channels by exploiting the hierarchical structure of the JPEG2000 bit stream. In all these cases, the possible benefits that may result also from applying an efficient MIMO transmission scheme are not taken into account. In [7], an UEP scheme in the spatial domain is proposed by making use of beam-forming algorithms and exploiting the frequency diversity present in a closed loop MIMOOFDM system.

Still, in most cases the channel state information is not available at the transmitter (CSIT) or if present it may not be well suited to the actual characteristics of the channel mainly due to the high user mobility and inherent feedback delay time. Thus, often it is impossible to take a real time adaptation decision. All of these factors impose a closer look to the benefits of open loop MIMO communications to systems that feature unequal error protection.

The issues concerning such systems require a more detailed exploration, yet some ideas can be found in [8-10]. In all these articles, schemes for providing unequal error protection both in the spatial domain (via space-time coding or spatial multiplexing) and in the time domain (via punctured convolutional codes or block turbo codes) are proposed. The idea is to assign a differentiated error protection level of the useful data, according to its importance, by exploiting the redundancy present in both domains. A higher level of protection will be assigned in one domain and a lower level in the other, such as to obtain a good trade-off between the transmission efficiency and reliability.

Still, a disadvantage of these schemes comes both from an insufficient exploration of the image content as well as from the usage of suboptimal MIMO transmission schemes. An efficient use of the available limited resources can be obtained only by properly matching the error protection level provided by the channel code to the degree of error sensitivity characterizing the useful data.

The rest of the paper is organized as follows: Section 2 is dedicated to a brief overview of the employed MIMO STBCs, while Section 3 is dedicated to the JPWL error protection mechanisms as well as to the way a UEP strategy can be applied based on the information contained in the JPWL markers. Section 4 is devoted to the description of the used system model while in Section 5 the obtained results are presented. Section 6 concludes the paper.

\section{MIMO STBCs}

Since high fidelity image transmission requires large bandwidth and reliability, MIMO systems prove to be extremely advantageous. Most of the research done in the field of MIMO space time coding techniques has focused on the design of space time codes, with the goal of minimizing the overall error rate, but without taking into account the properties of the source content [3].

In the current paper it will be shown that the error performances of a certain STC technique can be greatly improved by combining it with a suitable error control coding technique. The first STBCs were due to Alamouti [11], who discovered a remarkable transmission scheme able to offer a normalized rate of 1 symbol/s over two transmit antennas and two time periods, while maintaining a simple MLD (Maximum-Likelihood Decoding) at the receiver.

At each encoding operation, the space time encoder will take a block of two modulated symbols, $s_{1}$ and $s_{2}$ and map them on two transmit antennas, according to the codeword matrix expressed in Relation (1).

$$
C_{\text {Alamouti }}=\left[\begin{array}{cc}
S_{1} & -s_{2}^{*} \\
S_{2} & S_{1}^{*}
\end{array}\right]
$$

In [12], a new family of linear space time codes were proposed, the DAST (Diagonal Algebraic Space Time) codes, by making use of rotated constellations constructed based on the algebraic number field theory. The DAST codes provide full diversity for an arbitrary number of transmit antennas, as well as a normalized rate of 1 symbol/s. Also, they outperform the orthogonal design codes for complex constellations, when the number of transmit antennas is greater than two, but at the cost of an increased decoding complexity that can be greatly reduced by applying a sphere decoding algorithm.

The codeword matrix, for the case of a $2 \times 2$ MIMO system is presented in Relation (2), and it was obtained by spreading a rotated version of the information symbols $s_{1}$ and $s_{2}$ over the diagonal of a square matrix by the Hadamard transform. In Relation (2), $\left(x_{1}, x_{2}\right)^{T}=M_{n} \cdot\left(s_{1}, s_{2}\right)^{T}$ where with $M_{n}$ it was denoted the complex or real rotation matrix, and with $(\cdot)^{T}$ it was denoted the transpose of the matrix.

$$
C_{D A S T}=\left[\begin{array}{cc}
x_{1} & x_{2} \\
x_{1} & -x_{2}
\end{array}\right]
$$

Later, in [13] the Golden $2 \times 2$ perfect code was proposed, the term perfect denoting full diversity, full rate of $N_{T}$ symbols per channel use for a number of $N_{T}$ transmit 
antennas, as well as a non-vanishing determinant for an increased spectral efficiency and uniform average transmitted energy per antenna [3]. The name Golden comes from the usage of the Golden number, $\theta=(1+\sqrt{5}) / 2$ in its construction. The codeword matrix is shown in Relation (3), where with $s_{1}, s_{2}, s_{3}, s_{4}$ are denoted the information modulated symbols. Also $\bar{\theta}=1-\theta$,

$$
\begin{aligned}
& \alpha=1+i(1-\theta) \text { and } \bar{\alpha}=1+i(1-\bar{\theta}) \text {. } \\
& C_{\text {Golden }}=\frac{1}{\sqrt{5}} \cdot\left[\begin{array}{cc}
\alpha\left(s_{1}+s_{2} \theta\right) & \alpha\left(s_{3}+s_{4} \theta\right) \\
i \bar{\alpha}\left(s_{3}+s_{4} \bar{\theta}\right) & \bar{\alpha}\left(s_{1}+s_{2} \bar{\theta}\right)
\end{array}\right]
\end{aligned}
$$

Due to the remarkable properties of this code, both in [13] and [14] it was shown that such a code construction outperforms all previously known code constructions, especially for the medium SNR (Signal to Noise Ratio) range.

\section{JPWL Error Protection Mechanisms}

To overcome the limitations of the baseline JPEG2000 error resilience mechanisms, which prove to be insufficient as they do not correct transmission errors just detect them and conceal the erroneous data, four new marker segments have been added by JPWL. These are: EPC (Error Protection Capability), with the role of signaling the usage of other marker segments, EPB (Error Protection Block) that contains information related to the error detection and correction mechanisms employed, ESD (Error Sensitivity Descriptor) that contains information related to the degree of error sensitivity of the code-stream data and RED (Residual Error Descriptor) that is used for signaling the presence of residual errors in the code-stream [1].

As choosing a predefined error protection strategy for the whole image does not prove to be a good approach, as it leads to an inefficient use of the available resources, in the current paper a differentiated error protection scheme is proposed, by exploiting the information present in the EPB and ESD markers. For error correction, $R S(N, K)$ codes will be used, where $K$ bytes of useful information symbols are protected by $N-K$ bytes of redundant symbols, the error correction capability of the code being $(N-K) / 2$.

It is now proven that the code-stream header (Main Header and Tile Header) is the most sensitive part of the whole code-stream as the presence of even a single error in this part could lead to the impossibility of decoding the whole code-stream [2]. Thus, the default error correction codes, as specified in [1], RS $(160,64)$ and RS (80, 25) are applied for the protection of the Main Header, respectively Tile Header.

Further improvements can be obtained by applying an uniform level of protection (EEP) for the whole data packets that make up the image. The benefits that reside from protecting the image header, and mainly from applying a uniform protection of the whole code-stream data, are highlighted in Figure 1.

An Alamouti STBC was considered, and QPSK symbols were transmitted for testing the system's performance under the most robust conditions. The image quality is assessed in terms of the PSNR (Peak Signal to Noise Ratio) fidelity metric, based on a pixel by pixel comparison between the original image and the rebuilt image.

From Figure 1 it can be observed that in case of protecting even only the image header, an improvement of up to $5.58 \mathrm{~dB}$ in the PSNR can be obtained for a SNR of $6 \mathrm{~dB}$ with respect to the transmission of the unprotected JPEG2000 sequence. This is due to the increase of the image correct decoding probability, as the errors present in the image header often lead to a decoder crash. Significant PSNR improvements of up to $22.6 \mathrm{~dB}$ for an SNR of $10 \mathrm{~dB}$, compared with the unprotected JPEG2000 case, can be obtained by applying an equal error protection (EEP) of the whole code-stream data, even with the least robust RS $(37,32)$ code.

When applying an EEP technique, the contribution of all data packets to the overall image quality is considered to be equal, although as shown in [2] the JPEG2000 code-stream is organized hierarchically. Thus, the lower quality layers are more sensitive to transmission errors than the higher quality layers, a correct decoding of these layers, and implicitly a significant improvement of the image quality, can be obtained only if they are more heavily protected [4]. So, by applying a differentiated protection of the code-stream data (UEP) according to its importance, either on a layer basis or on a packet basis, significant improvements can be obtained.

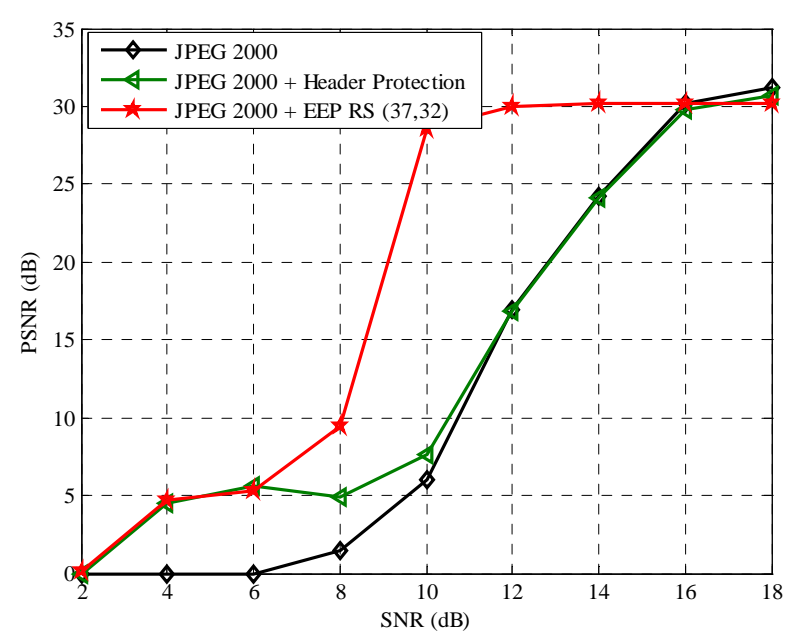

Figure 1. Highlight of the benefits that can be obtained by protecting the image header as well as by applying a uniform level of protection for the whole code-stream data. 
The way a UEP strategy can be implemented for protecting a three layered image, by making use of the EPB marker segments, is represented in Figure 2. The EPB0 data will be used for protecting the Main Header (SOC, SIZ and other marker segments) and the EPB1 data for protecting the Tile Header (SOT, other tile marker segments and SOD). The other EPB markers (EPB2, EPB3 and EPB4) are used for providing a differentiated protection of the compressed data packets according to their degree of error sensitivity.

\section{System Description}

The employed schematic is shown in Figure 3. A $2 \times 2$ MIMO system is considered, as this is the most used case in practice, where due to the limited size of receivers the mount of more than two receive antennas becomes a difficult task.

Based on the content to be transmitted, a joint source channel coding scheme is employed by making use of the JPWL error protection capabilities in order to assure the best tradeoff between the robustness to transmission errors and the perceived image quality. The incoming bits are then mapped onto a vector of symbols from a QPSK constellation. Then, the modulated symbols are grouped into blocks and transmitted both in space (over multiple antennas) as well as in time (over multiple time slots), according to some predefined codeword matrices.

A quasi-static flat Rayleigh fading channel model is considered, where the path gains are constant over the transmission of one frame (codeword interval) but may vary from one frame to another.

At the receiving side, the inverse process takes place; after the STC decoder and symbol demapper, a JPWL decoder is employed for correcting the errors and rebuilding the JPEG2000 frames.

\section{Simulation Results}

This section is dedicated to the presentation of the simulation results, obtained using the Matlab environment. The OpenJPEG simulator is used for encoding the $256 \times$ 256 color test image Lena.bmp, on four layers for the overall bit rates of $0.125 \mathrm{bpp}, 0.25 \mathrm{bpp}, 0.5 \mathrm{bpp}$ and 1 bpp. The average channel coding rate is maintained to 0.86 , to show the effectiveness of the proposed methods even for the case when the least robust error control codes are applied. All simulations were repeated 50 times for each SNR value, and the expressed results represent their average. Both an objective as well as a subjective image quality assessment is performed.

\subsection{STBCs Performance Analysis}

First of all, an analysis of the previously discussed STBCs

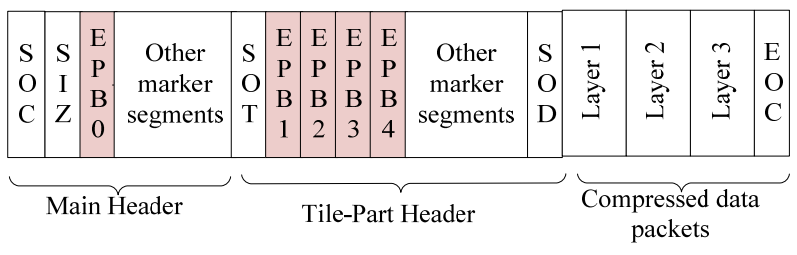

Figure 2. Applying EPBs to perform UEP.

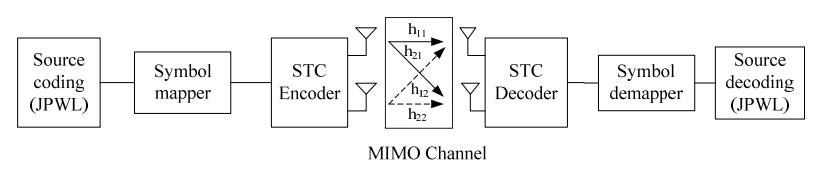

Figure 3. Proposed transmission scheme.

is performed upon an EEP RS $(37,32)$ sequence in the attempt to evaluate not only their BER (Bit Error Rate) performance but also the performance with respect to the content to be transmitted. The constellations of the employed STBCs have been adjusted to a common spectral efficiency of 4 bpcu.

As it can be observed from Figure $\mathbf{4}$ and it was confirmed also in [14], the Golden code outperforms the other employed STBCs. For a BER of $10^{-4}$ the SNR requirement is $5 \mathrm{~dB}$ less than for the case of the DAST code and approximately half of the previous value for the case of an Alamouti code. The better performances of the Golden code result from the construction of the codeword matrix, built with the aim of achieving an optimal DMT (Diversity-Multiplexing Trade-off) between the achievable data rate (link throughput) and diversity gain (link robustness). Thus, in case of the Golden code a number of four useful information symbols are transmitted during the duration of two time slots, as compared with Alamouti where only a half of these are transmitted in the same amount of time.

Among the three implemented STBCs, the DAST one proves to have the poorest error performance, as a result of the fact that these types of codes are quasi-optimal with respect to the coding gain [12]. The results in terms of PSNR for the three evaluated STBCs are presented in Figure 5, and are accompanied by subjective results in Figure 6.

Both from Figures 5 and $\mathbf{6}$ it can be observed that the choice of an optimal MIMO transmission algorithm can indeed lead to significant improvements with respect to the performance of a certain adopted error protection strategy. Still, the implementation of an optimal STBC is not all the times possible, mainly due to practical considerations as there isn't an optimal STBC for any number of transmit and receive antennas, and more than that, able to withstand different channel conditions.

In such a case, the performance of a STBC technique with respect to the perceived image quality can be enhanced by exploiting the source content and assigning a 


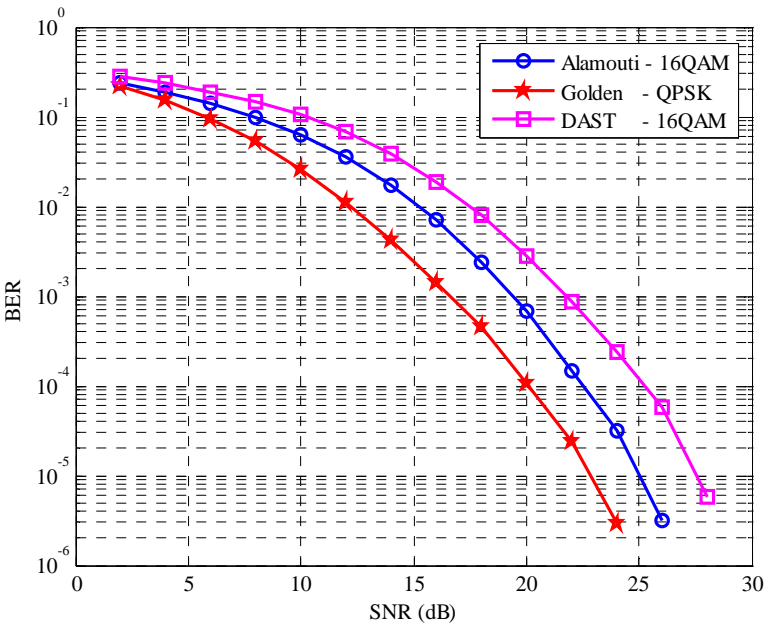

Figure 4. BER performance of different STBCs for a $2 \times 2$ MIMO system.

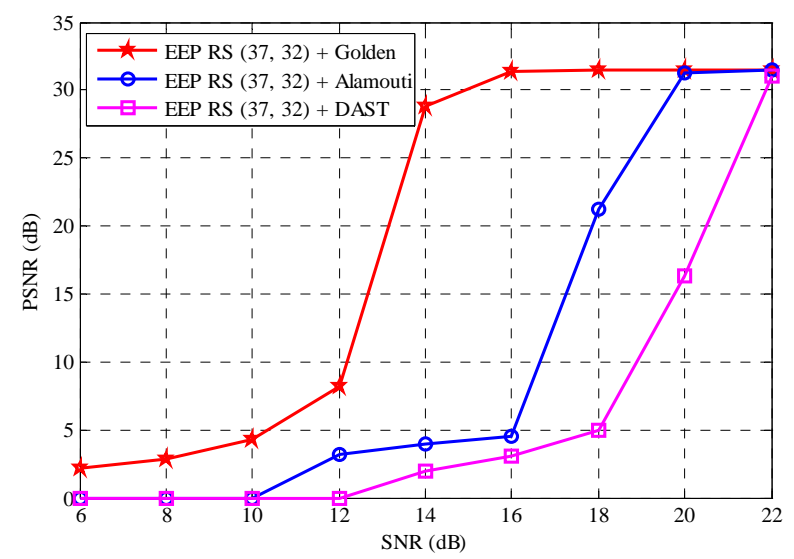

Figure 5. PSNR performance of different STBCs for a $2 \times 2$ MIMO system.

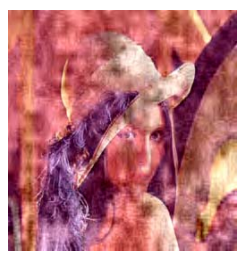

(a) EEP + DAST

PSNR $=16.72 \mathrm{~dB}$

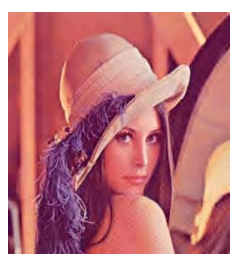

(b) EEP + Alamouti PSNR $=30.60 \mathrm{~dB}$

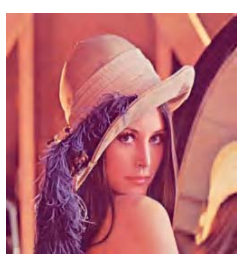

(c) EEP + Golden PSNR $=31.46 \mathrm{~dB}$
Figure 6. Subjective results of the decoded images for SNR $=20 \mathrm{~dB}$.

higher error protection scheme. This can be observed from Figure 7 where a lower PSNR performance, indicating a lower image quality, of the Alamouti code with respect to the optimal Golden code was compensated by applying a stronger level of protection by means of RS $(64,32)$. Still the price paid for this will consist in a lower image quality at high SNR values.

Furthermore, besides exploiting the information present in the EPB marker and implicitly the available RS codes, we will also exploit the data sensitivity to transmission errors by performing an analysis also on the ESD marker segment. In this way, we will try to improve even more the performance of the previous EEP RS $(37,32)$ strategy applied in conjunction with a Golden code.

\subsection{Analysis of the Proposed UEP Strategies}

Several unequal error protection (UEP) strategies will be proposed and compared with the classical equal error protection strategy (EEP) in the attempt to optimize even more the system's performance especially for low to medium SNR values. For this, a closer investigation of the data sensitivity to transmission errors is imposed.

A detailed analysis of the Lena.bmp image, in terms of the PSNR improvement obtained with the progression of the number of packets decoded from the image, leads us to the conclusion that a major PSNR improvement is associated to the decoding of the first four packets from the image. This can be observed both from Figure 8, where objective results are presented as well as from Figure 9, where subjective results are presented. Also, as observed from Figure 8, not all the data packets lead to a significant increase of the image quality, some being inserted only for preserving a symmetrical code-stream structure. Based on this idea, a selective packet error protection scheme is developed.

In the first UEP proposed strategy, the most important packets are considered to be from 1 - 4, 13 - 19 and 25 34 , and for these a higher error correction code is allocated, RS (64, 32). For the packets from 5 - 12 and 20 24 the same level of protection is assigned with the help of the RS $(37,32)$ code. The last packets from 35 - 48 will be transmitted without any protection, in order to compensate for the high level of protection allocated to the most significant packets.

The results obtained for this strategy are presented in Figure 10, but as observed they are modest, leading to a

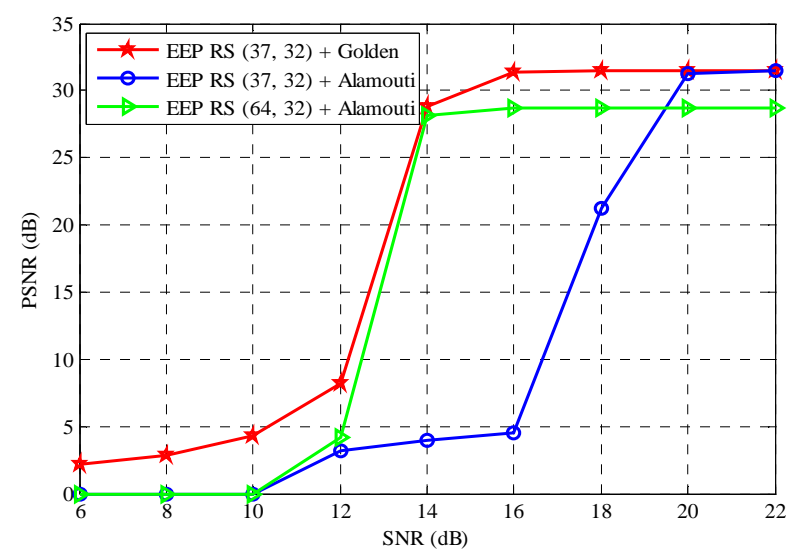

Figure 7. Improvement of the Alamouti PSNR performance by means of a higher error coding scheme. 


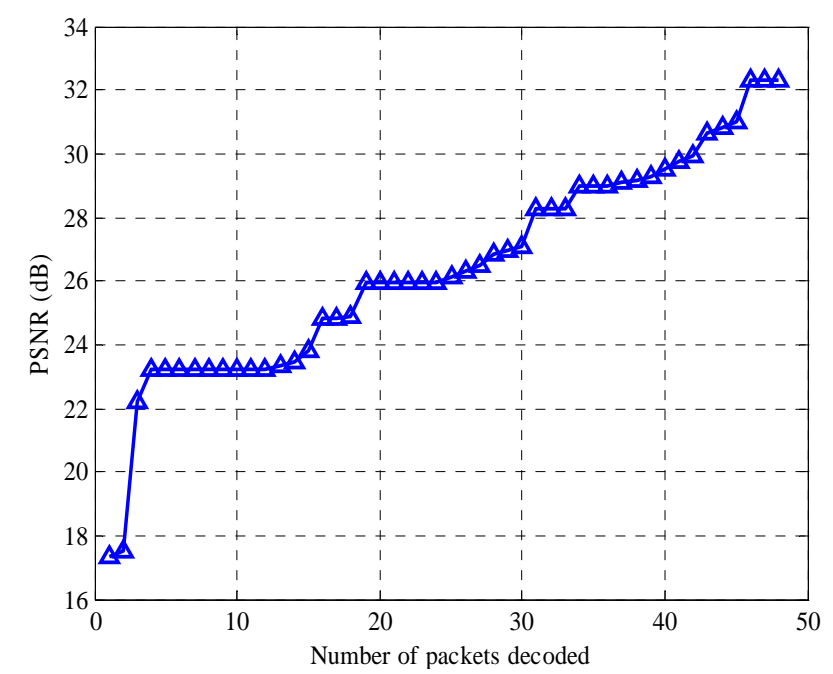

Figure 8. Progression of the average PSNR with the number of packets decoded from the image.

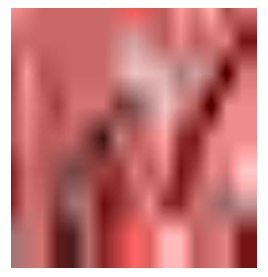

(a) two packets PSNR $=17.60 \mathrm{~dB}$

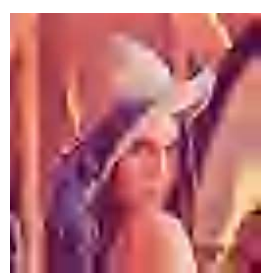

(b) three packets PSNR $=22.09 \mathrm{~dB}$

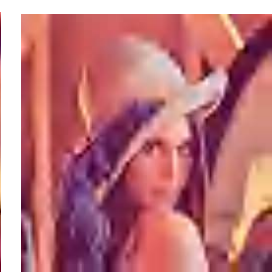

(c) four packets PSNR $=23.27 \mathrm{~dB}$
Figure 9. Visual results of the PSNR improvement obtained by decoding the first packets from the image.

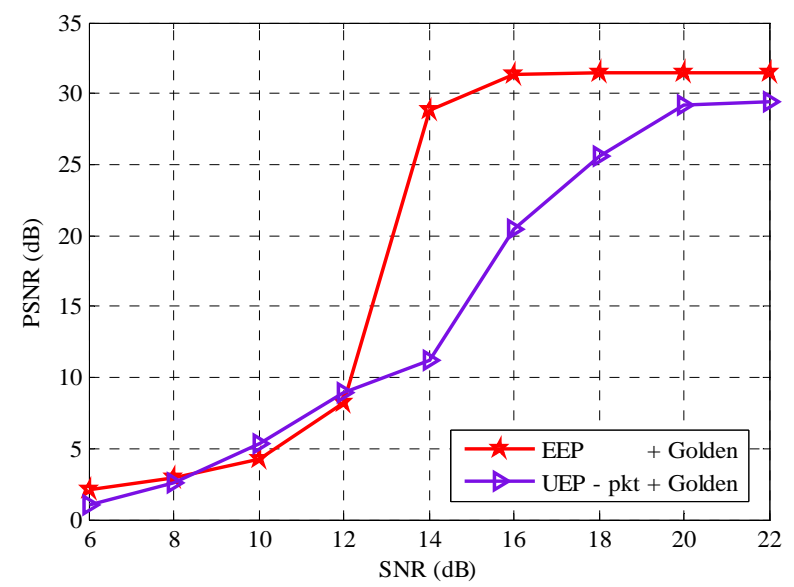

Figure 10. Comparison between EEP and UEP strategiespacket level.

PSNR improvement of at most $1.13 \mathrm{~dB}$ for an SNR of 10 $\mathrm{dB}$. This is due to the packet losses occurring as a result of unrecoverable errors that strongly affect the least protected packets, thus leading to a drop of that packet and eventually to a degraded image quality.

Rethinking the previous strategy, but this time at the layer level, with the intent to improve even more the qua- lity of the image especially at low SNR values leads us to the development of the two UEP strategies presented in Table 1. Since the information in the packets belonging to layer 1 (L1) is required to decode the information contained in layer 2 (L2) and so on, we can conclude that the sensitivity of data to corruption decreases as we move from a lower layer to a higher quality layer.

Thus, we have decided to assign all the available protection to the first three image layers, for obtaining at least a basic image quality of $29 \mathrm{~dB}$ out of $32.39 \mathrm{~dB}$ possible, and leave the last layer unprotected in order to balance for the high amount of protection allocated to the previous layers. Based on this idea, two UEP strategies both having the same overall correction capacity but differently distributed among the considered layers, are proposed and compared with the classical EEP strategy. In case of UEP1, a decreasing level of protection is considered for the three most important image layers, while in case of UEP2 the same amount of protection is evenly distributed among the three image layers.

The results obtained for the three proposed error protection strategies are presented in Figure 11. It can be observed that both UEP strategies outperform the EEP strategy for low to medium SNR values (6 - $12 \mathrm{~dB}$ ). The greatest PSNR improvement of $4.29 \mathrm{~dB}$ is obtained for an SNR of $10 \mathrm{~dB}$, for the case of the UEP2 strategy.

Also, what is interesting to be noted is that out of the two proposed UEP strategies the UEP1 strategy that considers a differentiated protection of the first three image layers offers an improved performance also for high SNR values as compared to UEP2, mainly due to the higher level of protection and correction allocated to the initial image layer. Thus, depending on the image content and channel conditions, especially at high SNR values, it may be preferable to assign less protection and transmit more image packets for an increased quality, rather than increase the protection level and discard useful packets.

In Figure 12, the PSNR performance versus BER is presented, in the attempt to characterize even more a certain applied UEP strategy. At a BER less than $10^{-3}$ the UEP and EEP strategies perform similarly, although the maximum UEP achieved quality (31.36 dB) is slightly less than the maximum quality achieved for the EEP case (31.46 dB). Between $10^{-3}$ and $10^{-2}$ the performance of

Table 1. Proposed layer EEP and UEP strategies.

\begin{tabular}{cccc}
\hline Layer No. & EEP & UEP 1 & UEP 2 \\
\hline L1 & RS (37, 32) & RS (51, 32) & RS (48, 32) \\
L2 & RS (37, 32) & RS (48, 32) & RS (48, 32) \\
L3 & RS (37, 32) & RS (45, 32) & RS (48, 32) \\
L4 & RS (37, 32) & 0 & 0 \\
\hline
\end{tabular}




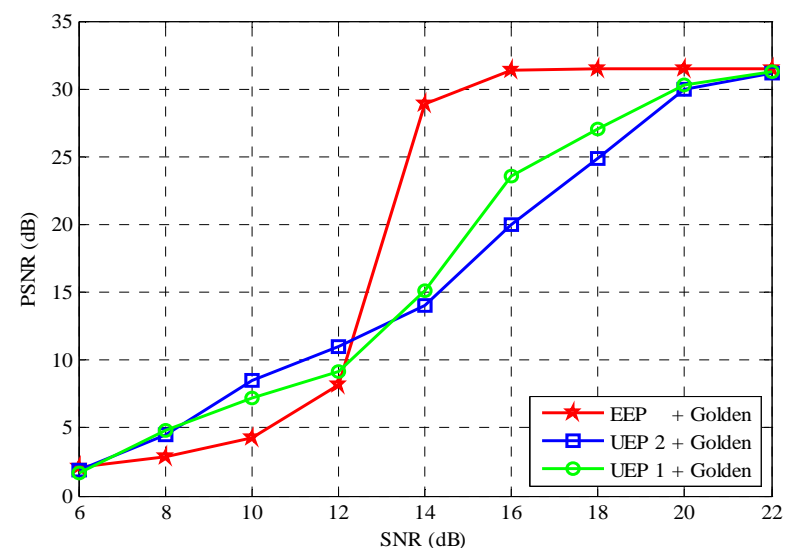

Figure 11. Comparison between EEP and UEP strategieslayer level.

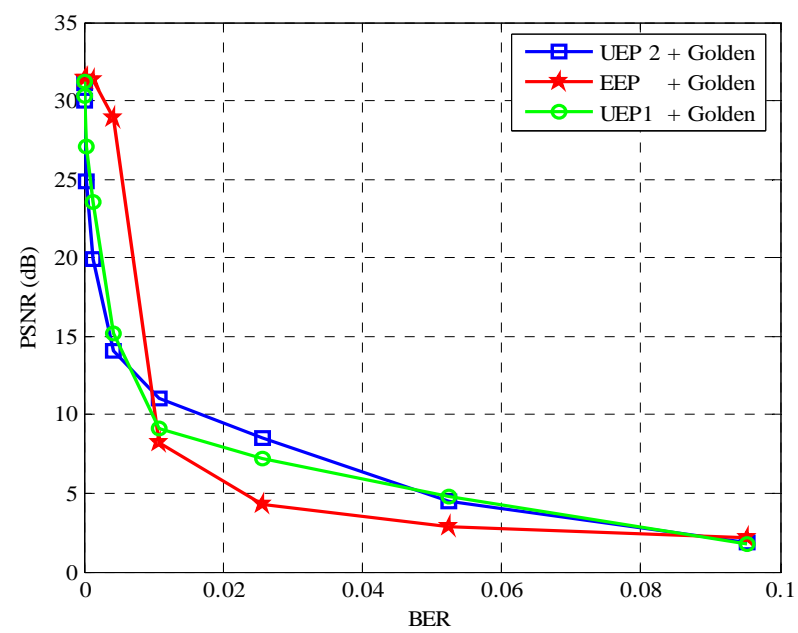

Figure 12. PSNR versus BER performance of the proposed UEP strategies compared to the EEP strategy.

both UEP strategies degrades due to the increase of the error rate present in the system as a result of the exceed of the correction capacity allocated to the superior image layers that are not so well protected. Still, with the continuously increase of the BER, the UEP strategies start to perform better mainly because of the higher protection assigned to the base layers, while in case of the EEP strategy once the correction capacity of the applied RS codes is overwhelmed, a rapid degradation of the image quality can be noted.

The performance degradation of the EEP strategy with respect to the PSNR metric, as a result of the drop of image correct decoding probability can be noted from Figures 13 and 14. As the packet losses induced by the channel may corrupt critical information necessary for the correct decoding of the whole image code-stream, at a certain point appears the risk of not being able to decode anymore the data packets. Thus, a decoder crash can appear especially once the error correction capabilities of the employed RS codes are exceeded, this lead-

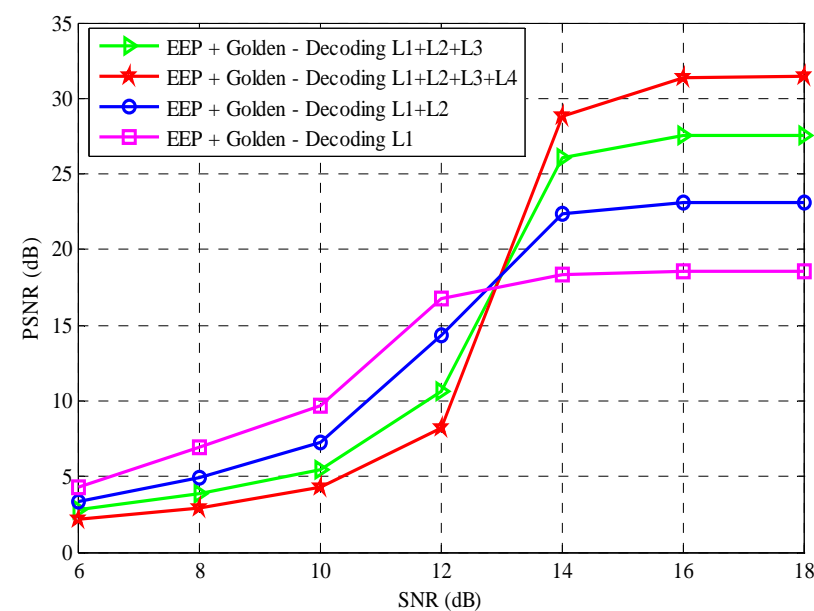

Figure 13. Degradation of the PSNR EEP performance with the increase of the number of image decoded layers $(L)$.

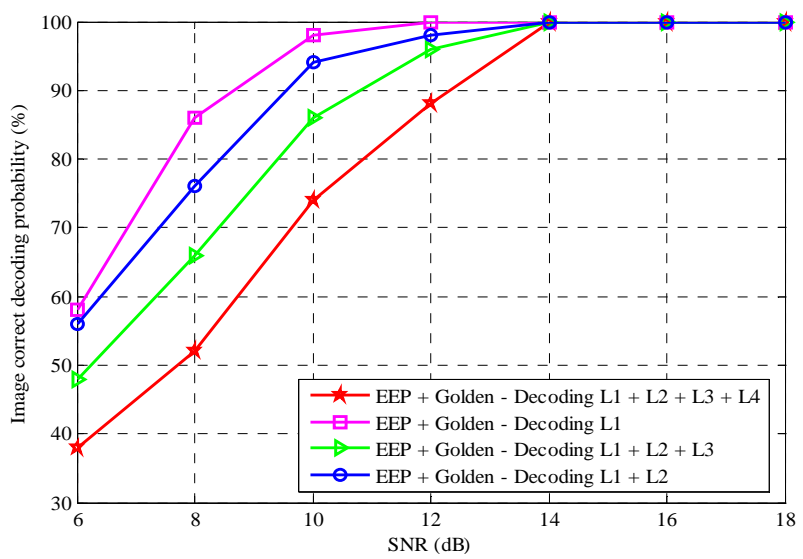

Figure 14. Degradation of the image correct decoding probability for the case of the EEP strategy with the increase of the number of image decoded layers $(L)$.

ing to an overall lower PSNR value.

Such an unwanted situation can be avoided by exploiting the channel state information available at the receiver, for the case of an open loop system. By imposing a threshold on the minimum acceptable image quality, it can be decided how many image layers are optimal to be decoded under certain channel conditions.

From the analysis performed upon the contribution of the data packets to the overall image quality, it was observed that a decoding of the packets belonging to the first three image layers leads to an acceptable visual quality, the decoding of the last image layer leading only to a small refinement of the whole image.

The results obtained for the case when only the first three image layers have been decoded are presented in Figures 15 and 16 and represent a preliminary work towards the design of a new MIMO STBC-UEP scheme where the optimal number of image layers to be decoded will be established dynamically, based on a continuous 


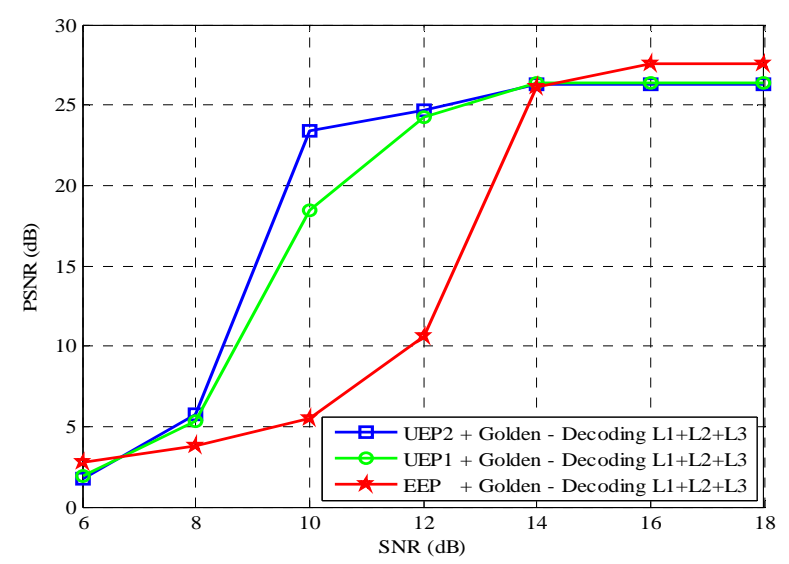

Figure 15. PSNR performance of the proposed UEP strategies in case of decoding only the first three image layers (L).

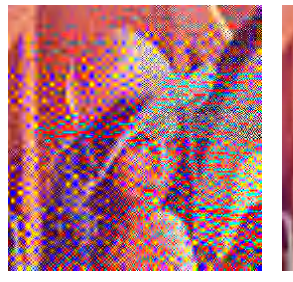

(a) EEP + Golden PSNR $=10.35 \mathrm{~dB}$

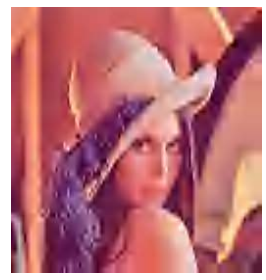

(b) UEP1 + Golden PSNR $=24.26 \mathrm{~dB}$

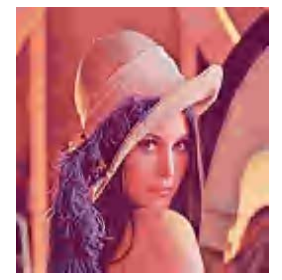

(c) UEP2 + Golden PSNR $=26.28 \mathrm{~dB}$
Figure 16. Visual results of the three considered strategies at a $\mathrm{SNR}=12 \mathrm{~dB}$.

monitoring of the transmission medium.

As it can be observed from Figure 15, by decoding only the first three image layers for the error protection strategies listed in Table 1, a more significant PSNR improvement of up to $17 \mathrm{~dB}$ with respect to the EEP case can be obtained, considering an SNR of $10 \mathrm{~dB}$ and the UEP2 strategy. This assumption holds also from a subjective point of view, as it can be noted in Figure 16.

\section{Conclusions}

The paper aims at showing the importance and the gain that can be obtained by exploiting the content to be transmitted in a MIMO OL system. Different strategies for optimizing the transmission of JPEG2000 coded images over a quasi-static Rayleigh fading channel were proposed. Better results can be obtained either by combining a lower coding gain spatial technique (ex: Alamouti) with a higher error protection scheme (ex: RS $(64,32)$ ) or by combining a higher coding gain spatial technique (ex: Golden) with a lower error protection scheme (ex: RS $(37,32)$ ), without inducing any overhead with respect to the total bandwidth or transmission power.

Furthermore, different UEP strategies were proposed with respect to the classical EEP strategy, in the attempt to optimize even more the performance of the system by exploiting the hierarchical structure of the JPEG2000 code-stream. As shown, an optimal UEP strategy that leads to the best performance for all channel conditions cannot be found, usually the use of such a technique being preferred for a low to medium SNR range. Also, in applying such a link adaptation technique, a compromise must always be made between the maximum level of protection added and the minimum quality that one wants to obtain even under a worst case scenario.

\section{Acknowledgements}

The presented work was supported by the French National Research Agency within the CAIMAN project, ANR-08-TO-002.

\section{REFERENCES}

[1] ISO/IEC 15444-11, "Information Technology_JPEG2000 Image Coding System. Part 11: JPEG2000 Wireless,” 2007.

[2] T. Holl, V. Lecuire and J.-M. Moureaux, "Error Sensitivity of JPEG2000 Codestream for Efficient Data Protection on Unreliable Networks," Proceedings of the 14th European Signal Processing Conference, Florence, 4-8 September 2006.

[3] V. K. Madisetti, "The Digital Signal Processing Handbook,” 2nd Edition, CRC Press, Boca Raton, 2010, pp. 27(1-16).

[4] C. Hao, Z. Ye and Z. Lei, “Application Method of JPEG2000 Wireless Standard,” 2nd IEEE Conference on Industrial Electronics and Applications, Harbin, 23-25 May 2007, pp. 2049-2053. doi:10.1109/ICIEA.2007.4318770

[5] V. S. Sanchez and M. K. Mandal, “Adaptive Unequal Channel Protection for JPEG2000 Images,” Proceedings of Indian Conference on Computer Vision, Graphics \& Image Processin, Ahmadabad, 16-18 December 2002.

[6] M. Agueh and H. Soude, “Optimal Layer-Based Unequal Error Protection for Robust JPEG2000 Images and Video Transmission over Wireless Channels," Proceedings of the First International Conference on Advances in Multimedia, Colmar, 20-25 July 2009, pp.104-109. doi:10.1109/MMEDIA.2009.26

[7] U. Sethakaset and S. Sumei, "Robust JPEG2000 Image Transmission over Closed-Loop MIMO-OFDM with Limited Feedback," The IEEE 19th International Symposium on Personal, Indoor and Mobile Communications, Cannes, 15-18 September 2008, pp. 1-5. doi:10.1109/PIMRC.2008.4699750

[8] M. F. Sabir, R. W. Heath and A. C. Bovik, “An Unequal Error Protection Scheme for Multiple Input Multiple Output Systems," Conference Record of the Thirty-Sixth Asilomar Conference on Signals, Systems and Computers, Vol. 1, 3-6 November 2002, pp. 575-579. doi:10.1109/ACSSC.2002.1197246

[9] Y. Wang, Y. Du, S. Yu, K. T. Chan and Y. Qiao, “An Adaptive UEP_BTC_STBC System for Robust H.264 Video Transmission,” IEEE International Conference on Multimedia and Expo, 6-8 July 2005, pp. 129-132. 


\section{doi:10.1109/ICME.2005.1521377}

[10] H. Zheng, C. Ru, L. Yu and C. W. Chen, "Robust Video Transmission over MIMO-OFDM System Using MDC and Space Time Codes," IEEE International Conference on Multimedia and Expo, Toronto, 9-12 July 2006, pp. 633-636. doi:10.1109/ICME.2006.262488

[11] S. M. Alamouti, “A Simple Transmit Diversity Technique for Wireless Communications,” IEEE Journal on Selected Areas in Communications, Vol. 16, No. 8, 1998, pp. 14511458. doi:10.1109/49.730453

[12] M. O. Damen, K. Abed-Meraim and J.-C. Belfiore, "Diagonal Algebraic Space-Time Block Codes,” IEEE Tran- sactions on Information Theory, Vol. 48, No. 3, 2002, pp. 628-636. doi:10.1109/18.985979

[13] J.-C. Belfiore, G. Rekaya and E. Viterbo, “The Golden Code: A 2 × 2 Full-Rate Space-Time Code with Nonvanishing Determinants," IEEE Transactions on Information Theory, Vol. 51, No. 4, 2005, pp. 1432-1436.

[14] P. Rabiei, N. Al-Dhahir and R. Calderbank, "New Rate-2 STBC Design for 2 TX with Reduced-Complexity Maximum Likelihood Decoding," IEEE Transactions on Wireless Communications, Vol. 8, No. 4, 2009, pp. 1803-1813. doi:10.1109/TWC.2009.071323 